Davide Moscato

Maria I. Peracchi

Giovanni Mazzotta

Lidia Savi

Pier Antonio Battistella

\section{Post-traumatic headache from moderate head injury}

Published online: 20 July 2005

D. Moscato (凶) • M.I. Peracchi • G. Mazzotta Headache Centre San Carlo IDI Rome, Via Aurelia 275, Rome, Italy

e-mail: d.moscato@idi.it

\section{G. Mazzotta}

Childhood Neuropsychiatry Chair, Perugia, Italy

L. Savi

Headache Centre,

Turin, Italy

P.A. Battistella

Headache Centre,

Padua, Italy

\begin{abstract}
The onset of post-traumatic headache occurs frequently in children, where it is often caused by severe head injuries, therefore, it is part of a post-traumatic syndrome, rather than of an independent headache and in this case no cause is clearly evident. The problem, conversely, arises in post-traumatic headache after a light trauma, since it is difficult to establish the cause-effect link. We have studied PTH incidence for one year in the patients of the emergency ward of the Saint Charles of Nancy

Hospital, compared to the activity of 4 Italian headache centres. At the Saint Charles of Nancy Hospital of
\end{abstract}

98 patients with PTH after a moderate head trauma, 18 had acute and 26 chronic $\mathrm{PTH}$, the majority ceased after six months. In the Italian headache centres 1656 patients were examined, of these $3.2 \%$ suffered from PTH: 25 acute, 29 chronic. These data confirm the poor evidence of PTH after a light trauma and lead to doubt of the existence of this nosological entity.

Key words Post-traumatic headache - Sleep disturbances • Trigger factor

\section{Introduction}

The onset of post-traumatic headache (PTH) in children occurs with an incidence between $30 \%$ and $70 \%[1,2]$. Headache is often caused by severe head injuries, and as such is accompanied by other symptoms (weakness, dizziness, nausea, difficulty concentrating, insomnia, etc.) and, therefore, it is part of a post-traumatic syndrome, rather than of an independent headache and in this case no cause is clearly evident.

The problem, conversely, arises in PTH after a light trauma, because it is difficult to establish the cause-effect link. Hence, the definition of tension-type headache (TTH) or migraine or post-traumatic tension headache.
Already in the past several authors had doubts about this entity: in the epidemiological works conducted by Bille [3], Sillanpaa [4], Raskin [5] and Lanzi [6], there was no difference in the characteristics of headache in subjects who had suffered from head injury and others in whom it had already appeared. Also, in 1975 Haas [7] suggested that the same pathogenic mechanism existed in PTH and migraine in young patients, however, that the trauma was only a triggering momentum. In 1993, the same author did not find any relation between the severity of a trauma and the onset of chronic headache, and confirmed this in 1997 [8]. The new classification gives very precise indications both for the onset $<7$ days after the trauma, and the duration $<3$ months for acute headache and $>3$ months for chronic headache. This is due to the 
fact that headache is also considered a consequence of the trauma and, as such, it must disappear together with the other consequences. In daily activity, it is often difficult to establish the exact time of onset, because if the head injury is moderate, there may be other more important symptoms.

\section{Materials and methods}

In order to understand the incidence of PTH after a mild head injury, we studied its incidence for one year from two privileged observatories: in the patients of the emergency ward of the $\mathrm{S}$. Carlo Hospital, compared to the activity of 4 Italian headache centres.

\section{Results}

At the S. Carlo Hospital, PTH symptoms were found in 810 cases of head injury and of these, 275 were aged 3-18 years; only 98 were the consequence of moderate/light head injury. The latter 98 cases $(66 \mathrm{M}, 32 \mathrm{~F})$ underwent follow up after one week and after 8 weeks, in order to evaluate the type of trauma, neurological examination, development of headache and other symptoms. All of them presented negative X-rays, MRI $(n=8)$ and neurological examination. The causes for the trauma were: car accidents $4.3 \%$, household accidents $41.3 \%$, school accidents $30.4 \%$, sports accidents $17.4 \%$ and aggression $6.6 \%$. The diagnoses were acute PTH (for 18 patients it looked like TTH); 26 chronic PTH (14 chronic daily headache), which in most of the 18 cases ceased after six months. There was a preceding migraine in 19 patients and episodic TTH in 13 patients. Associated symptoms were dizziness in 40, unstable humour in 62 , alternation of the sleep-wake rhythm in 19 and dysomnias in 20 .
In the other headache centres 1656 patients $(873 \mathrm{~F}$, $783 \mathrm{M})$ aged 4-18 years were examined for the first time. Of these, 3.2\% suffered from PTH, 25 (12 F, $13 \mathrm{M})$ from acute PTH and 29 (13 F, $16 \mathrm{M})$ from chronic PTH. The subsequent headache had developed as migraine without aura in 26, migraine with aura in 2, ETTH in 16 and $\mathrm{CDH}$ in 9. Twenty-five children had a family history of headache and 20 had already suffered from headache. The head injury was due to play in $70 \%$ and car accidents in $30 \%$.

Sleep disturbances, as can be seen in Figure 1, which were already present (sleep talking, sleep walking, nightmares, bruxism, difficulty falling asleep) increased by 20\%-30\%, and events of aggressiveness and panic crisis appeared in $20 \%$ of the patients; all of the above disturbances have almost reverted to the percentages preceding the trauma after some months have passed.

The presence of anxious expressions as well as the increase in dysomnias may be due to the reaction to the traumatic event; this is also confirmed by the higher presence of such disturbances in those patients in which the head injury is a consequence of car accidents, and they could also be due to the axonal microdamage. As such the temporary improvement may be due to two situations, on one hand the reassurance mechanism, and on the other, the healing of axonal damage.

\section{Conclusions}

These data confirm on one hand the poor evidence of PTH after a light trauma and on the other hand they lead us to doubt the existence of this nosological entity.

Therefore, the indication already identified in the past, that head injury is a triggering factor of an already existing headache, but which has not yet appeared, seems confirmed, i.e., that a light head injury is only a trigger factor for the history of headache.

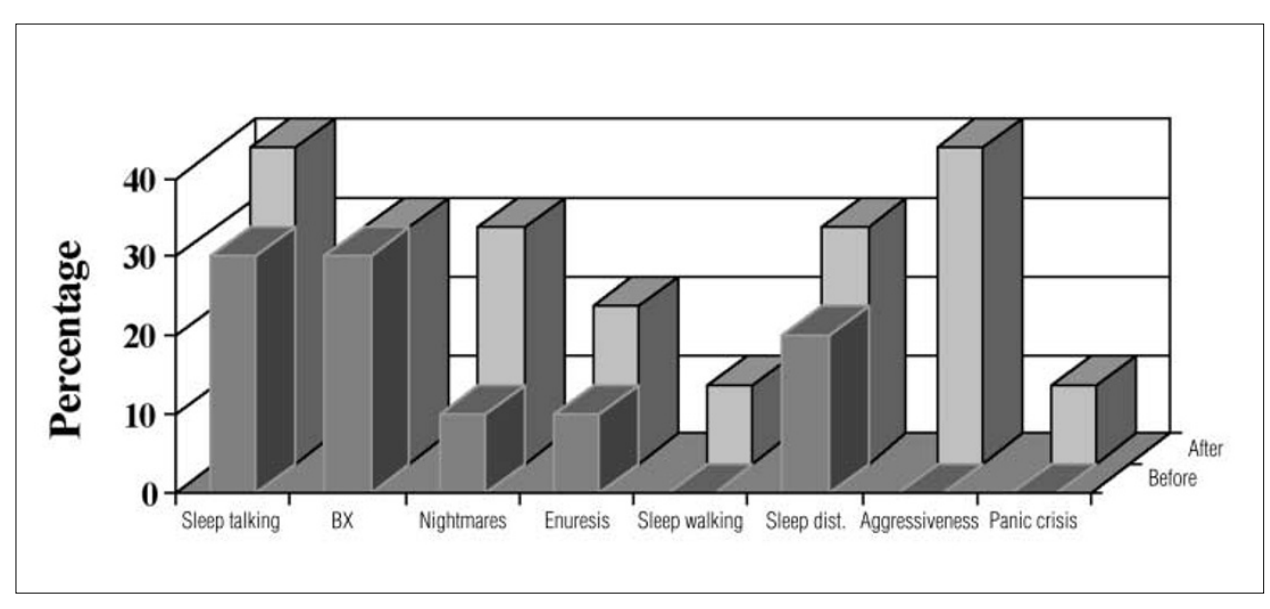

Fig. 1 Sleep disturbances in patients of headache centres 


\section{References}

1 Lance JW (1974) Mechanism and management of headache, 3rd edn. Butterworth, London, pp 227-233

2. Lanser JBK, Jennekins-Schinkel A, Peters ACB (1988) Headache after closed head injury in children.

Headache 28:229-230

3. Bille BO (1962) Migraine in school children. Acta Paediatr Scand 51[Suppl]136:14-151
4. Sillanpaa M, Piekkela P, Kero P (1991) Prevalence of headache at preschool age in an unselected child population. Cephalalgia 11:239-242

5. Raskin NH, Appenzeller O (1980) Post-traumatic headache. Headache Sanders Company 7:199-209

6. Lanzi G, Balottin U, Borgatti R, De Agostini G, Pezzotta S, Spanu G (1985) Late post-traumatic headache in pediatric age. Cephalalgia 5:211-215
7. Haas DC, Pineda GS, Lourie H (1975) Juvenile head trauma syndromes and their relationship to migraine. Arch Neurol 32:727-730

8. Haas DC (1997) Posttraumatic headache. Neurology 48:1735-1950 